\title{
Combinational use of lipid-based reagents for efficient transfection of primary fibroblasts and hepatoblasts
}

Kazuhiro Ishiguro, Osamu Watanabe, Masanao Nakamura, Takeshi Yamamura, Masanobu Matsushita, Hidemi Goto, and Yoshiki Hirooka

Department of Gastroenterology and Hepatology, Nagoya University

Graduate School of Medicine, Nagoya, Japan

BioTechniques 62:37-39 (July 2017) doi 10.2144/000114569

Keywords: transfection; primary fibroblasts; primary hepatoblasts

Supplementary material for this article is available at www.BioTechniques.com/article/114569.

Commercially available lipid-based transfection reagents are widely used to deliver DNA to cells. However, these lipid-based transfection reagents show poor gene transfer efficiency in primary cells. Here, we demonstrate a simple method to improve gene transfer efficiency in primary fibroblasts and hepatoblasts using a combination of lipid-based transfection reagents. Our data show that combined use of Lipofectamine LTX and FUGENE $\mathrm{HD}$ increases the efficiency of gene transfer compared with the use of either reagent alone, and this combination achieves the best result of any pairwise combination of Lipofectamine LTX, FuGENE HD, TransFectin, and Fibroblast Transfection Reagent.

Lipid-based transfection reagents are widely used for gene transfer in cells because they are commercially available and do not require special equipment. Moreover, transfection protocols using lipid-based reagents are simple and quite similar; according to the manufacturers' instructions for Lipofectamine LTX (ThermoFisher Scientific, Waltham, MA), FuGENE HD (Promega, Fitchburg, WI), TransFectin (Bio-Rad, Hercules, CA), and Fibroblast Transfection Reagent (Altogen, Las Vegas, NV), $0.5 \mu \mathrm{g}$ of DNA and $2 \mu \mathrm{l}$ of each reagent are separately diluted in $50 \mu \mathrm{l}$ serum-free medium, mixed, and incubated at room temperature; then, $300 \mu \mathrm{l}$ serum-supplemented medium is added. Finally, $400 \mu$ of the total mixture is used for transfection in each well of a 24-well culture dish. However, the lipid-based transfection method is inefficient for primary cells (1-3). Here, we demonstrate increased gene transfer efficiency in primary fibroblasts and hepatoblasts by the combined use of Lipofectamine LTX and FUGENE HD.

Primary fibroblasts were obtained from the skin of BALB/c mouse embryos on day 13.5 of gestation, as described previously (4). Primary hepatoblasts were obtained from the livers of ICR mouse embryos on day 13.5 of gestation, as described previously (5). Cells were maintained in DMEM containing 10\% FBS, $50 \mathrm{U} /$ $\mathrm{mL}$ penicillin $\mathrm{G}, 50 \mu \mathrm{g} / \mathrm{mL}$ streptomycin, and $125 \mathrm{ng} / \mathrm{mL}$ amphotericin $\mathrm{B}$. After 1-2 passages, cells were stored in liquid nitrogen and used within 5 passages after being thawed from the frozen stock.

pRL-CMV (Promega) is a plasmid that constitutively expresses Renilla luciferase under the control of the cytomegalovirus promoter. Luciferase activity has been used as an internal control representing gene transfection efficiency in each sample for the dualluciferase reporter assay system (Promega) (6). Therefore, pRL-CMV DNA was used to test transfection efficiency.

Supplementary Figure S1 shows the experimental protocol either for evaluating gene transfer efficiency by determining the activity of Renilla luciferase derived from $\mathrm{pRL}-\mathrm{CMV}$ or for assessing cell viability using the CellTiter-Glo luminescent cell viability assay (Promega). A Luminescencer PSN (model AB-2200-R; ATTO, Tokyo, Japan) was used to count relative light units (RLU), which reflected luciferase activity or cell viability. All experiments were performed in triplicate, and the data were presented as mean \pm SD. We used the Student's t-test for statistical analysis between the 2 groups and considered a $P$ value $<0.05$ to be statistically significant.

Our data showed the efficiency of gene transfer in primary fibroblasts was elevated by the combined use of $1 \mu \mathrm{l}$ Lipofectamine LTX and $1 \mu \mathrm{l}$ FUGENE HD, while reductions in cell viability were similar between use of the combined reagents and use of $2 \mu \mathrm{I}$ FuGENE HD alone (Figure 1A). Plus reagent is provided together with Lipofectamine LTX by ThermoFisher Scientific. As described in the manufacturer's instructions, the gene

\section{METHOD SUMMARY}

The Lipofectamine LTX and FuGENE HD lipid-based transfection reagents were used in combination to transfect primary fibroblasts and hepatoblasts, showing greatly increased efficiency of gene transfection relative to the use of either reagent alone. 
transfer efficiency of Lipofectamine LTX is increased by the addition of Plus reagent to diluted DNA in serumfree DMEM (Figure 1B). In our experiments, addition of Plus reagent also up-regulated the efficiency of gene transfer induced by transfection with a combination of Lipofectamine LTX and FuGENE HD (Figure 1B). Comparing pairwise combinations of transfection reagents, we confirmed that the best efficiency of gene transfer was achieved through the combined use of Lipofectamine LTX and FUGENE HD (Figure 1C).

We also tested the combination of Lipofectamine LTX and FuGENE HD for transfection of primary hepatoblasts. Although the efficiency of gene transfer in primary hepatoblasts was elevated by the reagent combination (Figure $2 \mathrm{~A}$ ), this increase was not as dramatic as that seen in primary fibroblasts (Figure 1A). We observed that the reagent combination reduced viability more than transfection with FuGENE HD alone in primary hepatoblasts (Figure 2A), unlike in primary fibroblasts (Figure 1A). According to manufacturers' instructions and previous reports $(7,8)$, cell density (cell confluency) can influence cytotoxicity and gene transfer efficiency when using lipidbased reagents. Cell density may affect cellular processes such as growth rate and endocytosis. Therefore, we tried the combination of Lipofectamine LTX and FuGENE HD for transfection under the different experimental conditions by increasing the density of primary hepatoblasts on Day -1 from $1 \times 10^{4}$ to $2 \times 10^{4}$ cells/well in a 96-well dish. Under these conditions, cell viability was minimally reduced, and gene transfer efficiency was further elevated by transfection with the reagent combination (Figure 2B).

Primary cells are more suitable for investigations into normal cellular physiology than transformed or immortalized cell lines since cell lines often show genetic and phenotypic differences from the cells that they originated from $(9,10)$. Although the transfer of foreign genes in cells is important for analyzing the biological functions of the genes, primary cells are difficult to transfect with DNA when using lipidbased reagents (1-3). Previous studies
A
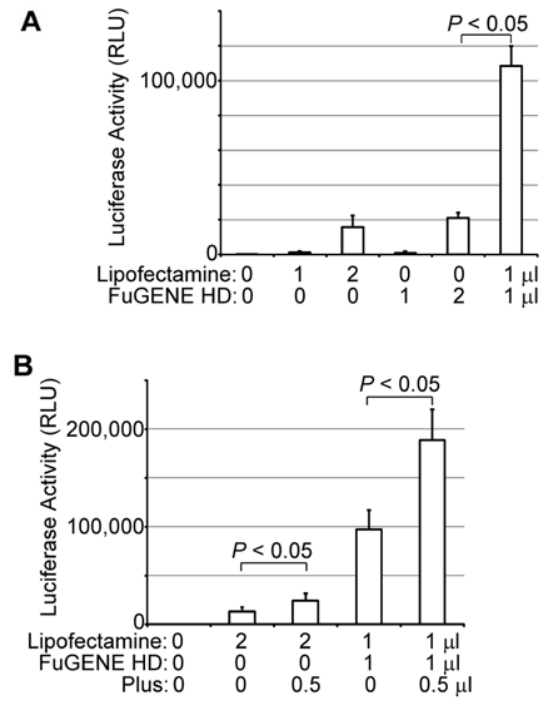

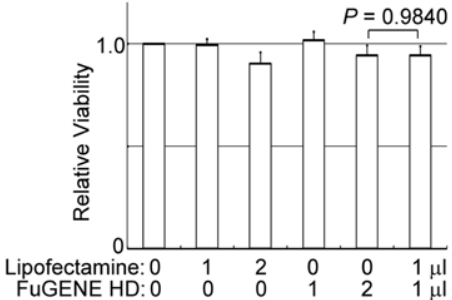

C

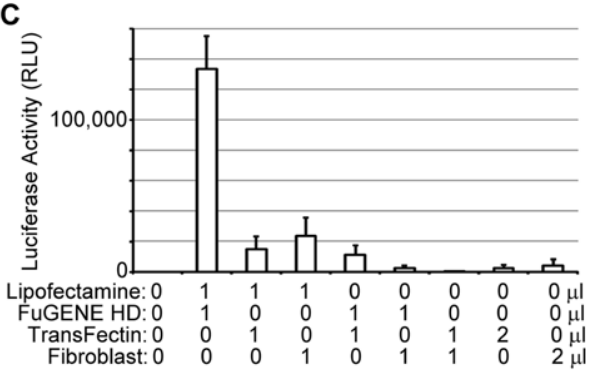

Figure 1. Effects of transfection of primary fibroblasts with different reagents. (A) pRL-CMV $(0.5 \mu \mathrm{g})$ was mixed with $0-2 \mu \mathrm{l}$ Lipofectamine LTX and/or FuGENE HD for transfection of primary fibroblasts. Renilla luciferase activity or cell viability was determined after 24 hours $(n=3)$. (B) pRL-CMV $(0.5 \mu \mathrm{g})$ was mixed with 0 or $0.5 \mu$ l Plus reagent and then with $0-2 \mu$ l Lipofectamine LTX alone or with FuGENE HD for transfection of primary fibroblasts. Renilla luciferase activity was determined after 24 hours $(n=3)$. (C) pRL-CMV $(0.5 \mu \mathrm{g})$ was mixed with $0-2 \mu \mathrm{L}$ Lipofectamine LTX, FuGENE HD, TransFectin or Fibroblast Transfection Reagent (in the amounts and combinations indicated on the $x$-axis) for transfection of primary fibroblasts. Renilla luciferase activity was determined after 24 hours $(n=3)$. $P$-values were determined using Student's $t$-test.

have presented several methods for gene transfer in primary cells, such as recombinant viral vectors, electroporation, and nucleofection (1,11,12). Gene delivery using viral vectors is efficient in most mammalian cell types, but viral vectors are randomly integrated into the host genome, resulting in undesired effects such as insertional mutagenesis (11). Moreover, the need to harvest recombinant viral vectors from packing cell lines takes substantial time and involves safety risks $(1,2,11)$. Electroporation and nucleofection (i.e., improved electroporation with cell type-specific solutions) use electrical pulses to create transient pores in plasma membranes (1,12). Although
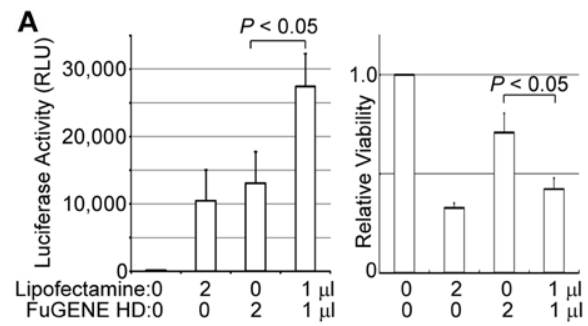

these methods can directly deliver DNA through the transient pores into cells, special devices are required to generate the electrical pulses $(1,12)$. The transfection protocol for this study uses a combination of two commercially available lipid-based reagents, Lipofectamine LTX and FUGENE HD, to increase the efficiency of gene transfer in primary fibroblasts and hepatoblasts without the need for any specialized equipment.

The use of a lipid/DNA complex for transfection was introduced by Felgner et al. (13): a single plasmid is surrounded by cationic lipids to neutralize the negative charge of DNA so that the lipid/DNA complex can

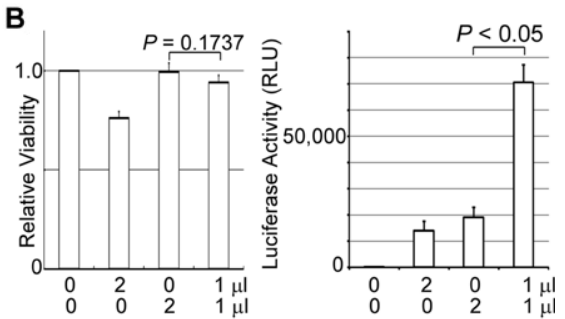

Figure 2. Effects of transfection of primary hepatoblasts with different reagents. On Day -1 primary hepatoblasts were seeded in wells of a 96-well dish: (A) $1 \times 10^{4}$ cells/well and (B) $2 \times$ $10^{4}$ cells/well. On Day $0,0.5 \mu \mathrm{g}$ of pRL-CMV was mixed with $0-2 \mu \mathrm{l}$ Lipofectamine LTX and/or FUGENE HD for transfection. Renilla luciferase activity or cell viability was determined 24 hours later $(n=3)$. $P$-values were determined using Student's $t$-test. 
associate with the negatively charged surfaces of cells. Lipid-mediated gene transfer depends on several factors such as net charge and the structure of the lipid/DNA complex (7). Lipid-based transfection reagents are composed of three domains: a positively charged head group, a hydrophobic region, and a linker that tethers the polar and non-polar regions. Formation of the lipid/DNA complex is a dynamic event because of the unstable interactions resulting from (i) the electrical attraction of DNA to the polar region of lipid; (ii) hydrophobic attraction of lipid to lipid via the non-polar region; and (iii) electrical repulsion of DNA by DNA $(7,14)$. Therefore, the lipid/DNA complex is presumed to form several different multilamellar lipid-DNA structures $(7,14)$. There is much interest in designing novel reagents carrying DNA or to improve the application of already available reagents for better gene transfection efficiency in a variety of cells by modifying the interactions between DNA and its carriers (15). We hypothesized that lipid/DNA complex formation may be optimized for transfection in primary fibroblasts and hepatoblasts using the combination of Lipofectamine LTX and FuGENE HD. However, the precise mechanism remains unknown because the chemical structures of these reagents have not been published by their manufacturers.

In conclusion, we present a simple method for efficient gene transfer in primary fibroblasts and hepatoblasts based on the combined use of Lipofectamine LTX and FUGENE HD. Addition of the Lipofectamine LTX Plus reagent is a useful option to further increase the efficiency of gene transfer. the Japan Society for the Promotion of Science [grant number $16 \mathrm{~K} 09306$ to K.I.].

\section{Competing interests}

All authors declare no conflict of interest.

\section{References}

1. Gresch, O. and L. Altrogge. 2012. Transfection of difficult-to-transfect primary mammalian cells. Methods Mol. Biol. 801:65-74.

2. Mellott, A.J., M.L. Forrest, and M.S. Detamore. 2013. Physical non-viral gene delivery methods for tissue engineering. Ann. Biomed. Eng. 41:446-468.

3. Horwood, N.J., C. Smith, E. Andreakos, E. Quattrocchi, F.M. Brennan, M. Feldmann, and B.M. Foxwell. 2002. High-efficiency gene transfer into nontransformed cells: utility for studying gene regulation and analysis of potential therapeutic targets. Arthritis Res. 4(Supp/3):S215-S225.

4. Ishiguro, K., K. Kadomatsu, T. Kojima, H. Muramatsu, S. Tsuzuki, E. Nakamura, K. Kusugami, H. Saito, and T. Muramatsu. 2000. Syndecan-4 deficiency impairs focal adhesion formation only under restricted conditions. J. Biol. Chem. 275:5249-5252.

5. He, Y., J.W. Zhou, L. Xu, M.J. Gong, T.C. $\mathrm{He}$, and Y. Bi. 2013. Comparison of proliferation and differentiation potential between mouse primary hepatocytes and embryonic hepatic progenitor cells in vitro. Int. J. Mol. Med. 32:476-484.

6. Xu, Y.Z., C. Kanagaratham, S. Jancik, and D. Radzioch. 2013. Promoter deletion analysis using a dual-luciferase reporter system. Methods Mol. Biol. 977:79-93.

7. Ma, B., S. Zhang, H. Jiang, B. Zhao, and H. Lv. 2007. Lipoplex morphologies and their influences on transfection efficiency in gene delivery. J. Control. Release 123:184-194.

8. Pinnaduwage, P., L. Schmitt, and L. Huang. 1989. Use of a quaternary ammonium detergent in liposome mediated DNA transfection of mouse L-cells. Biochim. Biophys. Acta 985:33-37.
9. Alge, C.S., S.M. Hauck, S.G. Priglinger, A. Kampik, and M. Ueffing. 2006. Differential protein profiling of primary versus immortalized human RPE cells identifies expression patterns associated with cytoskeletal remodeling and cell survival. J. Proteome Res. 5:862-878.

10. Pan, C., C. Kumar, S. Bohl, U. Klingmueller, and M. Mann. 2009. Comparative proteomic phenotyping of cell lines and primary cells to assess preservation of cell type-specific functions. Mol. Cell. Proteomics 8:443-450.

11. Thomas, C.E., A. Ehrhardt, and M.A. Kay. 2003. Progress and problems with the use of viral vectors for gene therapy. Nat. Rev. Genet. 4:346-358.

12. Colosimo, A., K.K. Goncz, A.R. Holmes, K. Kunzelmann, G. Novelli, R.W. Malone, M.J. Bennett, and D.C. Gruenert. 2000. Transfer and expression of foreign genes in mammalian cells. Biotechniques 29:314-324.

13. Felgner, P.L., T.R. Gadek, M. Holm, R. Roman, H.W. Chan, M. Wenz, J.P. Northrop, G.M. Ringold, and M. Danielsen. 1987. Lipofection: a highly efficient, lipidmediated DNA-transfection procedure. Proc. Natl. Acad. Sci. USA 84:7413-7417.

14. Dass, C.R. 2004. Lipoplex-mediated delivery of nucleic acids: factors affecting in vivo transfection. J. Mol. Med. (Berl.) 82:579-591.

15. Rezaee, M., R.K. Oskuee, H. Nassirli, and B. Malaekeh-Nikouei. 2016. Progress in the development of lipopolyplexes as efficient non-viral gene delivery systems. J. Control. Release 236:1-14.

Received 05 April 2017; accepted 25 May 2017.

Address correspondence to Kazuhiro Ishiguro, Department of Gastroenterology and Hepatology, Nagoya University Graduate School of Medicine, Tsurumai-cho 65, Showa-ku, Nagoya, Aichi, 4668550, Japan. E-mail: kio@med.nagoya-u.ac.jp

To purchase reprints of this article, contact: biotechniques@fosterprinting.com

\section{Author contributions}

This study was designed by K.I., H.G., and Y.H. The experiments using primary fibroblasts were performed by K.I., O.W., M.N., and T.Y. The experiments using primary hepatoblasts were performed by K.I. and M.M. K.I. wrote the paper. All authors approved the final manuscript.

\section{Acknowledgments}

We thank Ms. Moriyama for technical support. This work was supported by
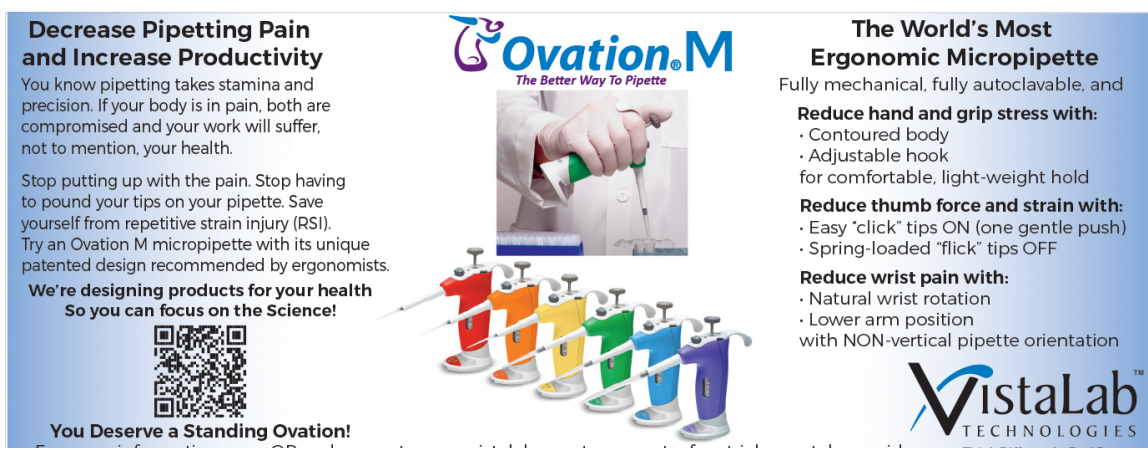